\title{
A CASE STUDY VALIDATION OF THE APPLICATION OF A GENERALISED EQUATION OF INNOVATION IN COMPLEX ADAPTIVE SYSTEMS
}

\author{
P. Malik ${ }^{1 * \#}$ \& L. Pretorius ${ }^{1}$
}

\section{ARTICLE INFO}

\begin{tabular}{ll}
$\begin{array}{l}\text { Article details } \\
\text { Submitted by authors }\end{array}$ & 22 Sep 2016 \\
$\begin{array}{l}\text { Accepted for publication } \\
\text { Available online }\end{array}$ & 14 Feb 2018 \\
& 31 May 2018 \\
\hline $\begin{array}{l}\text { Contact details } \\
\text { * Corresponding author } \\
\text { pravir.malik@deepordertechnologi } \\
\text { es.com }\end{array}$
\end{tabular}

Author affiliations

1 Graduate School of Technology Management, University of Pretoria, South Africa

\# $\quad$ The author was enrolled for a PhD (Technology Management) in the Graduate School of Technology Management, Department of Engineering and Technology Management, University of Pretoria

DOI

http://dx.doi.org/10.7166/29-1-1780
ABSTRACT

This article leverages a mathematical model of innovation for complex adaptive systems (CAS) to explore, ex post facto, organisational innovation investigations conducted by the first author at Stanford University Medical Center. Tapping into the meta-level and more varied sources of innovation, as illustrated by a generalised equation of innovation, requires that habitual patterns along the physical, vital, mental, and integral dimensions be overcome. The experimental work used frameworks and software consistent with the generalised equation of innovation in CAS to assist in overcoming such patterns at the personal, team, and departmental levels, thereby allowing deeper sources of innovation to come into being.

\section{OPSOMMING}

Hierdie artikel gebruik ' $n$ wiskundige model van innovasie bedoel vir ingewikkelde aanpasbare stelsels om, ex post facto, organisasiewye innovasie ondersoeke by Stanford University Medical Center te ondersoek. Deur gebruik te maak van die meta-vlak en meer gevarieerde bronne van innovasie, soos deur die veralgemeende innovasie-vergelyking geïllustreer, vereis dat gewoonte-patrone in die fisiese, lewensbelangrike-, verstandelike- en integraledimensies oorkom moet word. Die eksperimentele werk het gebruik gemaak van raamwerke en sagteware in ooreenstemming met die veralgemeende vergelyking van innovasie in ingewikkelde aanpasbare stelsels om sulke gewoonte-patrone op persoonlike-, span- en departementele vlakke te oorkom. Sodoende is dieper bronne van innovasie toegelaat om na vore te kom.

\section{INTRODUCTION AND RESEARCH APPROACH}

Innovation in complex adaptive systems (CAS) is often thought of as being emergent, paradoxical, unpredictable, randomly distributed, uncoordinated, and based on sensitive dependence on initial conditions $[7,9]$. Such a view is, perhaps, inevitable when CAS is viewed from the bottom up. But what if this point of view is changed to look at systems as a whole? This, in fact, may even be necessary from the point of view of broken symmetries. As Nobel Laureate Anderson suggests in his paper 'More Is different' [2], at higher levels of system complexity, existing symmetries are broken and new properties or behaviours that could never have been predicted by just studying the dynamics at a micro-scale come into being with the broken symmetries. When viewed from such a macro-level, innovation in CAS can be thought of as existing in every 'point-instant' of a CAS, and of having a cohesive mathematical framing [23], as summarised in Section 2.

Examples of CAS abound. In the natural world, brains, immune systems, ecologies, cells, developing embryos, and ant colonies; and in the human world, political parties, scientific communities, and the economy are examples of CAS [9]. Human knowledge itself has been suggested to be an example of CAS [33], as have living cities [15], for example. Considering complex corporate or organisational environments, the question is how innovation might be enhanced if it existed in every point-instant 
and had a cohesive mathematical framing [23]. This paper explores precisely this approach to enhancing innovation in a complex medical environment.

The first author of this paper was part of Stanford University Medical Center's Organizational Development group for five years until 2014. He was given permission to investigate, under the aegis of Mr Todd Prigge, the Director of the Department, and to attempt to bring about a higher degree of organisational innovation (as summarised in Sections 3 and 4), in part through the use of frameworks and software he had developed. Note that a key output and culmination of this work was a practical 'field guide', developed jointly by the primary author and Mr Prigge, that subsequently appeared in the book The Fractal Organization [22], and is briefly reviewed in Section 8. The frameworks and software used were consistent with an underlying mathematics of innovation for complex adaptive systems [23] as developed in the first author's doctoral work, and as summarised in part in Sections 3 and 4.

Note that this case study follows an ex post facto research approach to attempt to validate the efficacy of the organisational innovation framework as framed by the mathematical equations summarised in Section 3. Some of the attitudes, values, perceptions, and behaviours of individuals and groups at Stanford University Medical Center, as case study research, were interpreted over a multi-year period in the context of some of the mathematical equations summarised in Section 3. Note too that much of the data was collected in real-time during the years 2009 to 2013, using software tools that had already been developed and owned by the investigator, and as described in more detail in subsequent sections. A primary disadvantage of the ex post facto research approach is that the researcher, working in retrospect and therefore lacking control of the investigations already conducted, may be led to incorrect interpretation. However, given that the design of the software tools used during the years 2009 to 2013 was, in fact, consistent with the equations for innovation as described in Section 3, this disadvantage is minimised. The advantage of the ex post facto research approach is that a controlled inquiry can lead to useful interpretations in situations such as a complex real-time medical environment, where it is often difficult to conduct true investigations [28].

Section 3 reviews six key mathematical equations for innovation in CAS, developed as part of the doctoral research of the first author, and summarised in 'A mathematical basis of innovation' [23]. Section 4 highlights the essential methodology employed in bringing about a higher degree of innovation in an organisation. Sections 5,6 , and 7 review the approach used to overcome habitual physical, vital, mental, and integral patterns at the individual, team, and departmentalal levels respectively, aligned with Equations (1) to (6). Section 8 briefly reviews the practical field guide culmination of the work at Stanford University Medical Center. Section 9 offers a summary of the multi-year innovation investigation.

\section{MATHEMATICAL FRAMING OF INNOVATION IN COMPLEX ADAPTIVE SYSTEMS}

Reductionist thinking and linear modeling have ruled much of our practical problem-solving. By contrast, the emerging field of complex adaptive systems (CAS) is a way to understand the spontaneous, self-organising dynamics of the world. The body of CAS thinking has itself been heavily influenced by the work of Nobel laureates in multiple fields.

Chemistry Nobel laureate Ilya Prigogine, in his work on dissipative structures [27], showed these to be open systems that keep their order, and that may evolve towards a qualitatively different state as a result of the exchange of matter and energy with the external environment. Dissipative structures evolve through bifurcations and the alternation of continuity and discontinuity. As Prigogine explains, when the thermodynamic force acting on a system reaches high levels, it can cause the system to become discontinuous and to bifurcate in unexpected ways, creating an emergent property. In his work, Nobel laureate in physics Murray Gell-Mann [ ] asserts that the fundamental laws are quantum-mechanical. In his book The quark and the jaguar, Gell-Mann [13] explores the relationship between the simple and the complex. He asserts that quantum mechanics supplies only probabilities for alternative histories, and therefore chance must play a role in the unfolding of the universe. Nobel laureate in physics Richard Feynman, in his book QED: The strange theory of light and matter [12], states that the theory he presents will not explain why or how nature acts the way it does, but will explain with very high accuracy the probability that a photon emitted from a monochromatic light source is detected by a photon detector. Feynman jokingly goes 
on to suggest that the particles route could be absurd - going around Jupiter, to the local hot-dog stand, before reaching the detector. But this absurdity appears to have been generalised, and has become an edifice by which quantum nature is now framed and understood.

Building on the work of such luminaries, the world we live in is fundamentally projected as emergent, unexpected, evolving, uncertain, indeterministic, and random. The fundamental characteristics of the emerging field of CAS tend, too, to reflect these projections. But if the world were indeterministic and random, then chaos would likely be rampant and the world would be characterised by anarchy rather than by a general sense of progress. In several of the first author's books on fractals in complex systems, it is suggested that a pattern of progress emerges regardless of the area of consideration [20, 21, 22]. In his book, Does God play dice? [32], lan Stewart leverages Einstein's famous question to pose a new answer to this question by using the field of chaos. Einstein worried about quantum mechanics, which was generally held to be irreducibly probabilistic. Stewart asks: "Is it possible that the apparent randomness of the quantum world is actually deterministic chaos?" [32] Einstein himself stated: "The eternal mystery of the world is its comprehensibility... That the world is comprehensible is a miracle" [10]. In A brief history of time, Stephen Hawking makes the point that the fact that there are dualities in physics, or correspondences between apparently different theories of physics, suggests that there is a unified theory of physics that further indicates that the universe is governed by a set of rational laws that can be discovered and understood [17].

More recently, in his paper [35] and subsequent book [36] on a mathematics of life and civilization, Geoffrey West points out that the predominance of quarter-power scaling laws across all life forms is surprising, because each organism, sub-system, cell type, and genome has evolved in its own everchanging environmental niche within a unique history. For example, quarter-power refers to the phenomenon of requiring only a 75 per cent increase (multiple of a quarter) in metabolic energy when an organism increases in size. The emergence of systematic behaviour is what is surprising, and suggests that "generic underlying dynamical mechanisms have constrained evolutionary processes, thereby opening a possible window into determining quantifiable emergent laws that capture the essential features and coarse-grained behaviours of living systems" [35].

The question is, how deep and far does the systematic behaviour go? West has explored an aspect of the systematic behaviour as it relates to inputs required by systems as they increase in size, for a range of biological life-forms and the development of large cities. In this paper and the research on which it is based [25], the range of systematic behaviour explored has to do with what keeps systems complex, adaptive, and innovative. Furthermore, it is supposed that the systematic behaviour exists regardless of scale. A systematic mathematics was developed to frame this in the first author's doctoral work [25]. Key attributes of this mathematical theory for innovation in CAS include the following:

- This approach is inherently 'outside-in' focused, based on system-patterns and system-trends observed in space and time in general, thereby also integrating aspects of the existing 'bottomup' approach to CAS into it. The bottom-up approach is characteristic of key CAS schools of thought, such as MIT and Santa Fe Institute, among others. In these schools of thought, the characteristic of emergence is what creates top-down schema [9]. Thus, as suggested by GellMann, a complex adaptive system interacts with the environment, creates schemata (which are compressed and generalised regularities experienced in those interactions), behaves in ways consistent with these schemata, and incorporates feedback from the environment to modify and adapt its schemata for greater success [14]. The outside-in approach has been the focus of the first author's previous research, and is elaborated on in a series of books: Connecting inner power with global change [20], Redesigning the stock market [21], and The fractal organization [22].

- Building on Stewart's 'deterministic chaos' hypothesis [32], CAS is characterised by qualified determinism at each level of organisational complexity.

- $\quad$ This qualified determinism is orchestrated by a cohesive mathematical framework [26].

- This mathematical framework explains the spectrum of possibility from stagnation to sustainability / progress.

- The mathematical framework and the derived equations thus also provide a basis for innovation. 
- The mathematical framework advances the field of innovation by creating a series of mathematical equations to understand innovation better.

- The mathematical framework for innovation applies to organisations at different levels of complexity, from the 'simple' to the more 'complex'. Hence it will provide insight into how innovation happens at micro-levels, such as at the atomic / quantum-particle level and the level of the biological cell; at intermediate levels, such as at the level of the human being and the level of a team; and at more macro-levels, such as at the level of the corporation and the level of the market, among other levels.

- The mathematical framework provides insight into further potential development at each level of organisational complexity.

- This framework separates the nature of functionality by meta-layers, and suggests key dynamics that are operative at each layer. Under certain conditions, the respective meta-level functionality and operations become active, to bring about complexity and adaptiveness in the visible, surface layer.

- The mathematics of innovation suggested in this research constructs a generalised equation of innovation that may exist at multiple-levels of complexity, ranging from the micro- to the macro-levels. The mathematics constructs functions that highlight key operations for each relevant layer, and the interaction between layers, to bring about the emergent adaptability and complexity visible in the surface layer.

The generalised equation of innovation, introduced in Section 3, summarises several key attributes of this mathematical framework. The 'emotional intelligence builder tool' introduced in Section 5 (which was used as the primary means of data-collection at Stanford University Medical Center) has been designed to facilitate the connection of the several layers of innovation inherent in the aforementioned mathematical framework, and as summarised by the generalised equation of innovation.

\section{THE OBJECTIVES AND THE EQUATIONS}

The objective of the ex post facto case research work at Stanford University Medical Center - to engender a higher degree of organisational innovation - is summarised by the generalised equation of innovation [23], Equation (1), which depicts the conditions by which deeper sources of innovation are made active.

Equation (1):

$$
\text { Innovation }_{\text {orientation }-x}=\left[\begin{array}{c}
M_{3} \rightarrow \text { System }_{X} \\
(\uparrow F \rightarrow I) \\
M_{2} \rightarrow S_{\text {System }} \\
(\uparrow \text { Sig } \rightarrow F) \\
M_{1} \rightarrow \text { Sig }_{x} \\
\left(\uparrow>P_{x)}\right. \\
U \rightarrow x_{U}
\end{array}\right] \text { TC } \rightarrow x_{T}, \text { where }\left[\begin{array}{c}
x_{U} \ni[\ldots] \\
x_{T} \ni[\ldots]
\end{array}\right]
$$

Layers $U, M 1, M 2$, and $M 3$ may be thought of as the dynamic sources of innovation. According to the key attributes of the mathematical theory laid out in Section 2, the equation essentially postulates multiple sources of innovation in the 'core-matrix' (the vertical stacked lines that essentially highlight the dynamics of each source of innovation, and the condition required to get to the next higher vertical level in the matrix). The essential method to increase innovation in organisations is thus to shift the source of innovation to a higher level in the core-matrix.

The default layer is the surface or untransformed layer, $\mathrm{U}$; and the hypothesis is that organisations, regardless of size or complexity, tend to operate at this layer, which becomes the source of innovation for an organisation. As seen in Equation (1), the dynamics at this layer are typified by the following sub-equation:

$U \rightarrow x_{U}$.

$x_{U}$ is the untransformed set, where $x \ni[$ physical, vital, mental, integral $]$. 
As defined in 'A mathematical basis of innovation' [23]:

Physical $_{U} \ni[$ inertia, lethargy, status quo,...],

Vital $_{U} \ni[$ aggression, self centeredness, exploitation, ...],

Mental $_{U} \ni[$ fixation, fundamentalism, fragmentation, ... ], and

Integral $_{U} \ni[$ possession, usurpation, hidden agendas, ... ]

In other words, the sources of system innovation derive from untransformed sets that would, by definition, hardly be enduring and sustainable. The action of Equation (1) is to transform the untransformed sets, $x_{U}$, into the transformed sets, $x_{T}$. TC is the transformation circle function [23] that specifies the rate at which such transformation may proceed. A discussion of the remaining symbols in the equation follows.

It is possible, though, for the untransformed patterns $\left(P_{x}\right)$ to be overcome $(\uparrow>)$, as signified by the sub-equation:

$\uparrow>P_{x}$.

When this begins to happen, the dynamics of meta-level $1, M_{1}$ become more active $\left(\rightarrow \operatorname{Sig}_{x}\right)$, as per the sub-equation:

$M_{1} \rightarrow \operatorname{Sig}_{x}$

In this case, the dynamics are governed more by the generalised signature equation [23], Equation (2):

Sig $=X a+\overline{Y b_{0-n}}$ where $\left[\begin{array}{c}X \in\left[S_{\text {System }_{P r}}, S_{\text {System }_{P}}, S_{\text {System }_{K}}, S_{\text {System }_{N}}\right] \\ Y \in\left[S_{\text {System }_{P r}}, S_{\text {System }_{P}}, S_{\text {System }_{K}}, S_{\text {System }_{N}}\right] \\ a, b \text { are integers } ; a>b\end{array}\right]$.

As per the equation, there are then a large number of positive and unique sources of innovation that can become active, as represented by the sets $S_{\text {System }_{P r}}, S_{\text {System }_{P}}, S_{\text {System }_{K}}$, and $S_{\text {System }_{N}}$. The sets [23] are summarised below.

Set of System Presence:

$S_{\text {System }_{P r}} \ni[$ Service, Perfection, Diligence, Perseverance, ... ]

Set of System Power:

$S_{\text {System }_{P}} \ni[$ Power, Courage, Adventure, Justice, ...]

Set of System Knowledge:

$S_{\text {System }_{K}} \ni$ [Knowledge,Wisdom, Law Making, Spread of Knowledge ...]

Set of System Nurturing:

$S_{\text {System }_{N}} \ni$ [Love,Compassion, Harmony, Relationship ...]

Note that $\overline{Y b_{0-n}}$ signifies that the $\mathrm{Y}$-element can occur repeated times, in contrast to the X-element, which can occur only once. Note too, that the $X$-element has a higher weightage a than the weightage $b$ of any of the Y-elements. 
As can be observed, the nature of the sets at $U$ and $M_{1}$ are of a very different nature, with the sets at $M_{1}$ allowing for far more sustainable sources of innovation than $U$. When the signatures at $M_{1}$ become strong or become a force $(\mathrm{F})$, then the conditions for the dynamics of $M_{2}$ to become active are put in place. The signature becoming strong is represented by the sub-equation:

$\uparrow \operatorname{Sig} \rightarrow F$.

The dynamics of $M_{2}$ are signified by the sub-equation:

$M_{2} \rightarrow S_{\text {System }_{X}}$

In this case, it is the primary sets of architectural forces $\left(S_{\text {System }_{X}}\right)$ that become active [23]. Note that, in contrast to $M_{1}$, where it is some combination of the elements of the sets that becomes active, at $M_{2}$ it is the elements themselves that become active. Action at $M_{2}$ is therefore considered to be more enduring [26].

When the forces themselves become 'integral' (I), then the dynamics of $M_{3}$ can influence the untransformed layer $\mathrm{U}$ as well. The forces become integral, as represented by the sub-equation:

$\uparrow F \rightarrow I$.

The dynamics of $M_{3}$ are represented by the sub-equation below, where System ${ }_{X}$ represents the four-fold system-intelligence [23] concentrated at a point:

$M_{3} \rightarrow$ System $_{X}$.

By default, it is generally the untransformed layer $U$ that is active first. Activation of the other layers requires overcoming habitual patterns; this was the thrust of much of the work at Stanford discussed in the case study presented in this paper. The levers for overcoming the habitual patterns increase with the activation of the meta-layers along each dimension of the being, represented by the equations for the physical (Equation (3)), the vital (Equation (4)), the mental (Equation (5)), and the integral (Equation (6)). Equation (3), for instance, depicts the conditions by which deeper sources of innovation along the physical dimension are made active.

Hence, Equation (3):

Physical

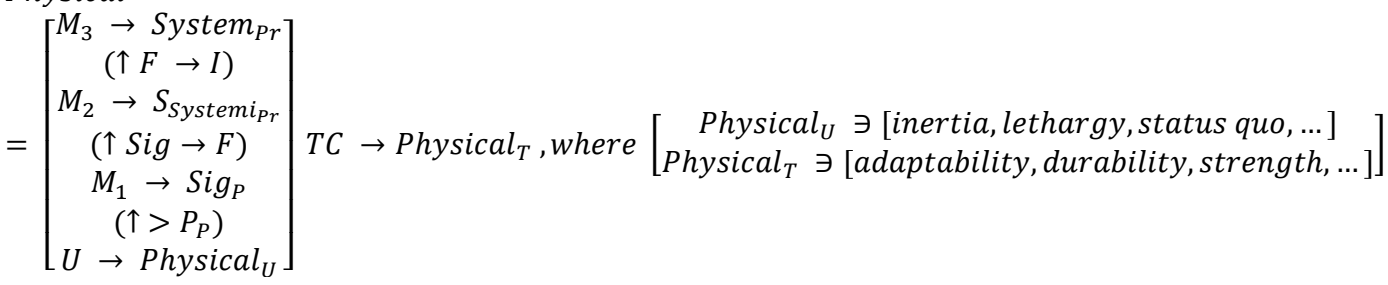

Equation (4):

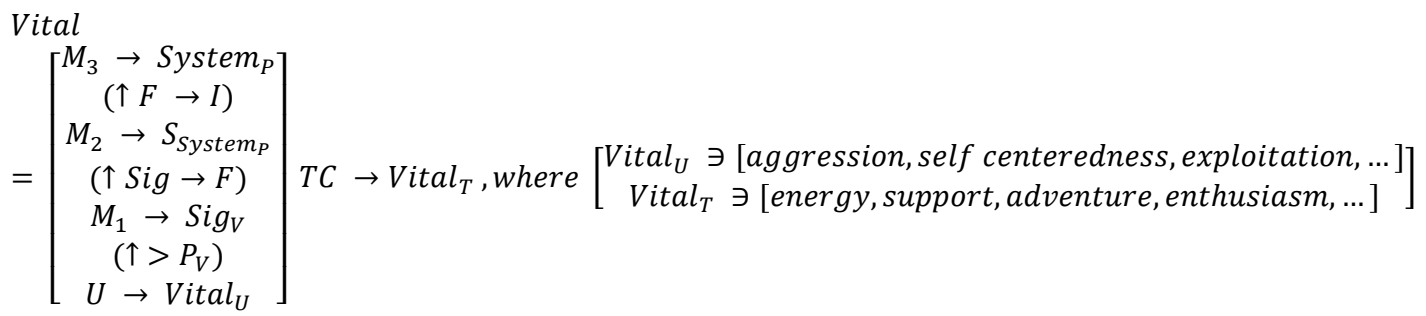


Equation (5):

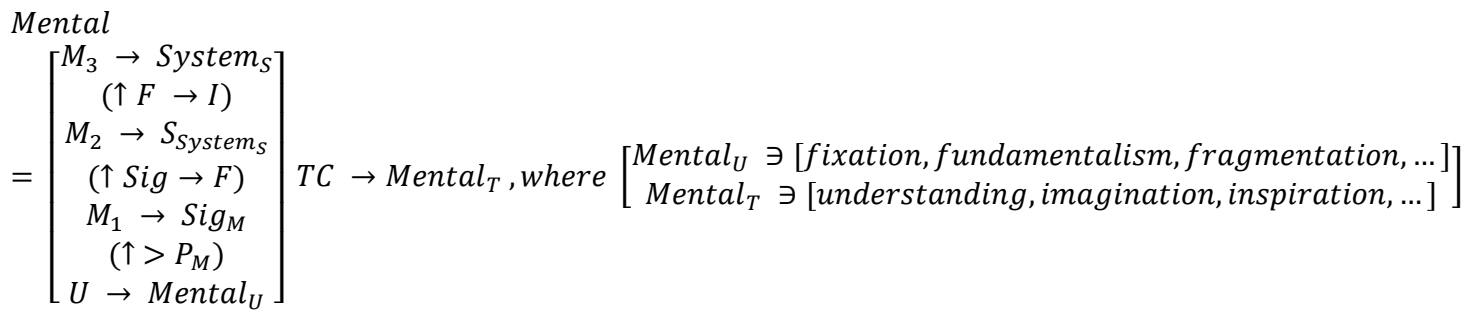

Equation (6):

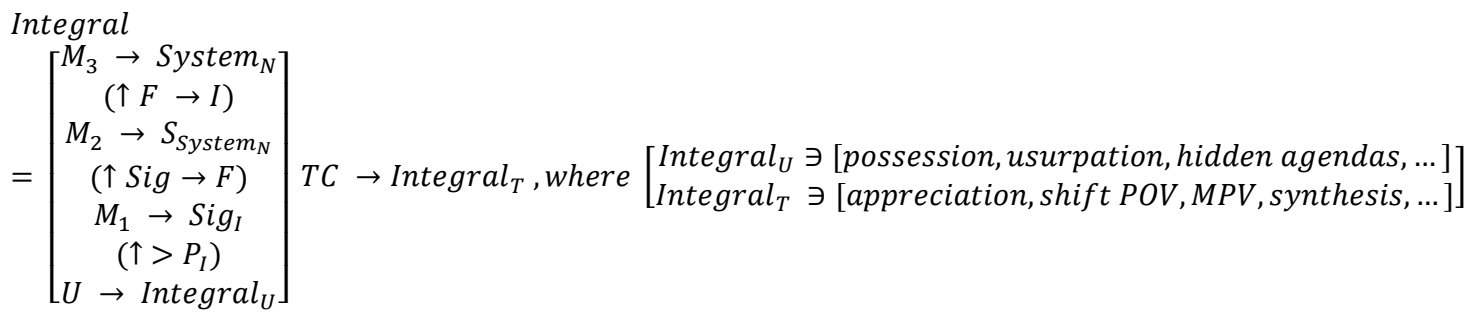

Basically, Physical $\mathrm{P}_{U}$ has to be progressively replaced by Physical $_{T}$; Vital $_{U}$ has to be progressively replaced by $\mathrm{Vital}_{T} ;$ Mental $_{U}$ progressively replaced by Mental $_{T}$; and Integral I $_{U}$ by Integral ${ }_{T}$.

The approach to increase innovation in an organisation is thus to move the active source of innovation from layer $U$ to the highest layer possible. This is done through fulfilling the sub-equation specified between the source-lines $\mathrm{U}, M_{1}, M_{2}$, and $M_{3}$.

To summarise: the aim of the work represented in this case study was to get employees, teams, and departments to overcome habitual patterns at their respective levels to allow access to deeper founts of innovation, as defined by the equations for the physical, the vital, the mental, and the integral, thereby allowing a greater range of sources of innovation to come into being. While it is difficult to measure such a vast range of possible sources of innovation, a single source in Section 6, synergy, belonging to the set of nurturing, $S_{\text {System }_{N}}$, was made through a controlled investigation as an illustrative example for this paper.

The emotional intelligence builder tool, introduced in Section 5, was the essential tool used actively to shift the source of innovation in the organisation. And since large organisations generally comprise departments, which comprise teams, which comprise individuals, another key aspect of the methodology to bring about larger-scale shifts in innovation is to start at the individual level, and then work through the team level to the departmental level. While Sections 5, 6, and 7 highlight such approaches respectively, Section 4 summarises key aspects of the methodology to increase innovation in an organisation.

\section{SUMMARY OF THE METHODOLOGY}

This section will focus on some practical aspects of mobilising equations (1) to (6), and the general methodology to increase innovation in an organisation.

Equations (1) to (6), introduced in the previous section, summarise the essential approach to increasing innovation in an organisation. Note that these equations are fractal, in that they can be applied to organisations of increasing scale and complexity. Thus they can be applied equally to individuals, teams, departments, and an organisation as a whole. 
In applying these equations, the following is to be kept in mind:

1. Equation (1), the generalised equation of innovation, summarises the overall approach to increasing innovation. It is a statement of the general dynamics that will be operative at each level of innovation, and of the general category of patterns that need to be overcome in reaching into, or activating, the next 'higher' level of innovation.

2. Equations (3) to (6) are instances of Equation (1), and summarise more specifically the dynamics and patterns particular to a way of being.

3. Equation (3) is an instance of Equation (1) along the 'physical' dimension. There are specific kinds of patterns to do with experience as a 'physical' being. The world-view that emanates from anchoring in this way of being can be both a block and an opportunity to further mobilising 'physical' sources of innovation. Equation (3) summarises the journey to freely activating innovation along the physical way of being.

4. Equation (4) is an instance of Equation (1) along the 'vital' dimension. There are specific kinds of patterns to do with experience as a 'vital' being. The world-view that emanates from anchoring in this way of being can be both a block and an opportunity to further mobilising 'vital' sources of innovation. Equation (4) summarises the journey to freely activating innovation along the vital way of being.

5. Equation (5) is an instance of Equation (1) along the 'mental' dimension. There are specific kinds of patterns to do with experience as a 'mental' being. The world-view that emanates from anchoring in this way of being can be both a block and an opportunity to further mobilising 'mental' sources of innovation. Equation (5) summarises the journey to freely activating innovation along the mental way of being.

6. Equation (6) is an instance of Equation (1) along the 'integral' dimension. There are specific kinds of patterns to do with experience as an 'integral' being. The world-view that emanates from anchoring in this way of being can be both a block and an opportunity to further mobilising 'integral' sources of innovation. Equation (6) summarises the journey to freely activating innovation along the integral way of being.

7. Equation (2) summarises the dynamics at $M_{1}$, where signature or uniqueness dynamics dominate. As can be seen in Equation (2), seeds are created through the combination of elements from deeper sets. Generally, these can only become active when habitual patterns at the surface layer, $\mathrm{U}$, are overcome.

Keeping these six equations in mind, the general methodology to increasing innovation in an organisation is further elaborated as the following:

1. Introduction to fractal theory and the power of patterns through the study of a fractal primer. This allows grounding in the work ahead. (Some description is given in Section 5.)

2. An initial facilitated session with a small group of people to introduce the emotional intelligence builder online tool (EQ tool). (Some description is given in Section 5.).

3. Commitment by individuals to use the $24 \times 7 \mathrm{EQ}$ tool to log states of being as they become aware of them.

4. Exercising of choice by individuals consciously to choose an alternative state of being, and logging these actions as they occur.

5. Awareness of alternative 'positive' patterns as they arise, and logging of these in the EQ tool.

6. Increasing identification by individuals of 'set(s)' with which positive pattern(s) may be associated.

7. At a threshold moment, begin actively to engage more teams in preceding steps 1 to 6 . (Some description is given in Section 6.)

8. Begin to work with more and more departments. (Some description is given in Section 7.)

9. The fractal effects of working with individuals, teams, and departments will begin to be felt at more complex levels of the organisation, and even in the way an organisation goes to market.

10. Use of the field guide as a systematic way further to increase innovation on a larger scale. (Some description is given in Section 8.)

\section{THE BEGINNING OF THE WORK - THE INDIVIDUAL LEVEL}

At the start of the ex post facto research journey, the core organisational group in the case study organisation, referred to as the organisational development (OD) group / team, studied the theory of fractal organisations that frames in words what is happening in the process of innovation, as 
captured by the mathematical equations summarised in Section 2. This was done through a detailed study of the organisational fractal primer, Connecting inner power with global change [20]. Equations (1) to (6), summarised in Section 2 to frame the work at Stanford, are fractal in nature, in that they frame the workings of innovation of CAS at any scale. They also suggest that the nature of CAS, at a level of complexity as dictated by the set of equations that frame the kernel of innovation, will influence CAS at a subsequent level of complexity, as implied by Equation (7), where ' $x$ ' is one of the dimensions of being, as summarised by Equations (3) to (6), and ' $n$ ' is the level of complexity. Thus Equation (7) depicts the fractal repercussion of an orientation on layers adjacent to it:

\section{Fractal: Orientation ${ }_{x} @$ Complexity $_{n} \leftrightarrow$ Orientation $_{x} @$ Complexity $_{n+1}$}

Following the study circle, the OD team (consisting of six people) went through a half-day workshop on the Emotional Intelligence Builder software [8]. This software helps one become aware of some habitual physical, vital, and mental emotion-based patterns to which people and teams are easily subject. Note that many of the elements in the untransformed sets at layer $U$ referred to in Equations (1) and (3) to (6) are accompanied by such physical, vital, and mental emotions that are bound up with the elements. The Emotional Intelligence Builder software focuses on the management of these emotions and, therefore, of the untransformed elements specified by the $X_{U}$ untransformed sets that often will not allow a person to be objective if they are seized by an emotion. Daniel Goleman refers to this phenomenon as 'amygdala hijack' in his book on emotional intelligence [16].

Such awareness creates the possibility of choice, as one may more consciously choose to invest in another response when the restricting pattern under consideration arises. The moment-by-moment choice-making facilitates the movement from the untransformed sets $X_{U}$ to the transformed sets $X_{T}$, which is the implicit goal of Equations (1) and (3) to (6). The six columns in Figure 1 below summarise the menu of habitual physical, vital, and mental emotional patterns available through the Emotional Intelligence Builder software.

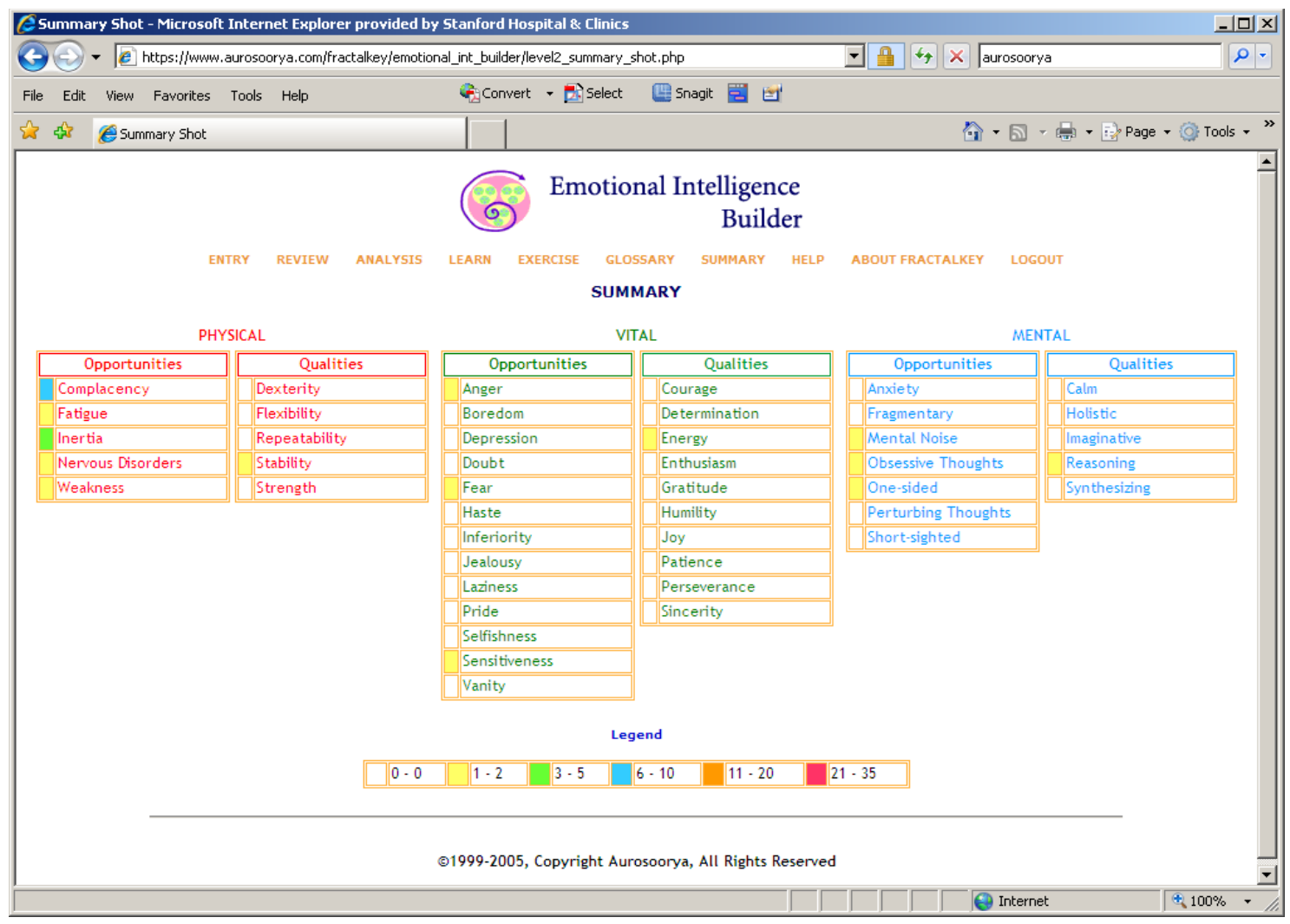

Figure 1: Summary of states of being (see online version for colour) 
Following the OD team's exposure to the software in facilitating the movement from $X_{U}$ to $X_{T}$ (as laid out in Equations (1) and (3) to (6), discussed in the previous section), the next logical step was to have a wider audience within Stanford experiment with the software. So the next stream of work, at the recommendation of Mr Prigge, took place with the Stanford Center of Education and Professional Development (SCEPD). Several custom courses that hinged on the Emotional Intelligence Builder software were developed, and were offered primarily to nurses at Stanford University Medical Center. One such course was in the use of technology to reduce stress, as captured by Figure 2.

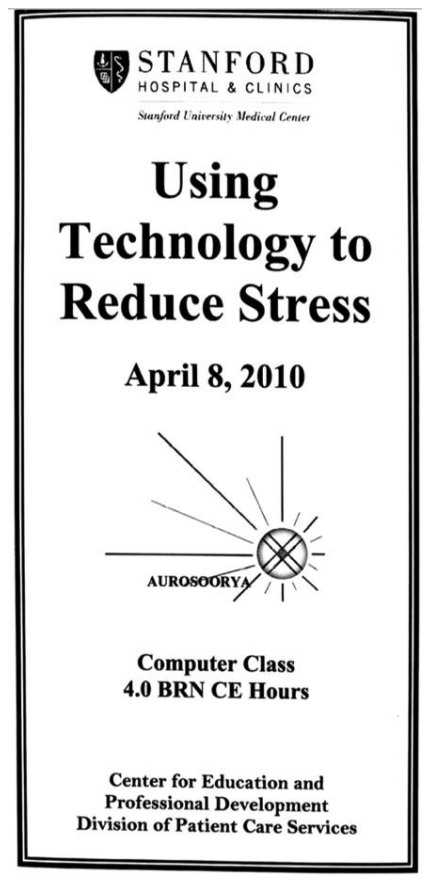

Figure 2: Course on using technology to reduce stress

These initial investigations resulted in the launch of a larger programme that focused on a core leadership group at Stanford Hospital \& Clinics.

\section{WORK AT STANFORD HOSPITAL \& CLINICS' LEADERSHIP ACADEMY - THE TEAM LEVEL}

There is value in holding up a mirror to teams, as suggested by Lencioni's The five dysfunctions of a team [19]. Planning and tracking plans, whether tasks completed, milestones met, or objectives fulfilled, are part of this, but only capture a fraction of the image. As important is the capturing of softer, feelings-based team dynamics, as covered by the Emotional Intelligence Builder software: What makes team members excited, or complacent, or fearful, or angry? What is the intensity of a feeling? What team dynamics may have caused it? Did a feeling persist beyond the boundary of a team meeting, perhaps keeping the team-member up at night? It is often the nature of persistent feelings that, over time, will make or break a team. Figure 3 illustrates the Emotional Intelligence Builder software instructions used in this research, which is aimed at capturing such 'softer' dynamics.

Equations (1) and (3) to (6) suggest that the nature of $U$ can be transformed by influences from the meta-levels $M_{N}$, where $\mathrm{N}=1,2$, or 3 . The questions in the instructions in Figure 3 assist individuals, teams, and departments to uncover how such transformation may be expressing itself. This transformation can be assessed by the changing nature of the patterns being captured for the case study in the Emotional Intelligence Builder software. Such a change in the nature of patterns may indicate the phenomenon of neuroplasticity, where connections between neurons are changed, bringing about change in response patterns given the same stimuli [6]. 
ENTRY allows observations relating to states of being to be entered into the diary. One state is entered at a time, and this can be repeated until all significant observations are recorded.

The state to be entered is selected from the appropriate column. If logging entries as part of a team, please keep the following core questions in mind. Note that these questions do not need to be exhaustively answered for all meetings. However, these questions serve as joggers, and the most significant ones can be answered. The determination of what to answer is up to you and will depend on the time you have available. Each team member should individually and independently enter information. Any resulting duplication of information from multiple perspectives will help to establish underlying and more significant patterns that the team may be experiencing:

1. How do team members respond to pressure?

2. How do members get along with one another? What is the level of tolerance between team members?

3. Are there serious issues and feelings that have been avoided? Are real issues being addressed by the team?

4. What is the level of confrontation? How does this show up on the team?

5. What leadership patterns have emerged?

6. Does the team agree on a single goal and a mutual plan?

7. What is the nature and result of conflict? How is conflict managed?

8. How are decisions made?

Note, that it is also possible, once you have established a sense for 'states of being' that arise in a meeting, to enter these without reference to the questions.

The INTENSITY bar allows the appropriate intensity of the state to be recorded. The range is from 1 to 10 , with 1 being 'low' and 10 being 'high'. You are encouraged to enter a value, though it is optional.

The DURATION bar allows the duration the state was active to be recorded. The range is from 5 minutes to 300 minutes. You are encouraged to enter a value, though it is optional. Note that typically higher values, beyond typical meeting times, indicate that a state of being may be becoming persistent.

The EVENT box allows the associated event accompanying the state to be recorded. You are encouraged to enter text. You can get as detailed as you want in these entries. You can cross-reference these entries to the questions listed above. Please get as nuanced as you need to, to describe what happened. Note that when entering data as a member of a team, these entries will remain hidden from normal view in the REVIEW module until such time as the team elects to have all entries be displayed. the team will then inform the system administrator to switch this viewing capability on.

The ADD ENTRY button actually records all the data on the form into the diary. This button should be selected only after an observation has been completely entered.

The RESET button clears all entered values.

The LEARN button allows the user to learn more about the specific state being entered. This is done by opening the LEARN module in a separate window. After the user has read the related information he or she can exit from that module and return to the ENTRY module.

The HELP button in the Master Menu on top, displays this help file.

The Date field defaults to the date of entry. It is not necessary to change the date field as it is understood that entries entered on the date will refer to past meetings till the current date. You could reference individual meetings in the EVENT box if you want to.

Figure 3: Emotional Intelligence Builder software instructions

Given the initial success with the OD team and with the courses offered through SCEPD discussed here as ex post facto case research, this web-based team-development software was used by Stanford Hospital \& Clinics to accelerate team development through its six-month Leadership Academy programme. The web-based tool computes fractal patterns in real-time and illustrates the deeper, and often unstated, dynamics occurring at the level of teams. The results obtained from this Stanford programme are used ex post facto as research data in this paper to reinforce the further efficacy of Equation (1), the generalised equation of innovation, and therefore of Equations (3) to (6).

Insight into fractal patterns is important because it is the 'small' behaviours, attitudes, and perceptions at the individual or team level that correlate with, and often determine, larger outcomes at the team, unit, and corporate level [22]. The Leadership Academy was an annual programme in the case study organisation in which close to 50 leaders from across the institute participated to develop and put into practice key leadership skills identified as critical to the organisation's future. Leaders were placed into teams that worked together on strategic projects.

The nature of the patterns that each team member experienced, or that each team member perceived other team members as experiencing, was self-captured in the web-based tool, 
anonymously or openly, depending on how transparent a team chose to be. Team members had to pause to really get a feel for what they were experiencing, and it is likely that such a pause and self-examination began to build a set of muscles not often used [11], by virtue of which a sustainable awareness field continues to grow in richness.

Tracking such information may shed light on the culture of a team in the process of being created. Focusing on feelings is critical, because failure to do so erodes the team environment and accelerates destructive dynamics. Successfully managing potentially destructive feelings, on the other hand, allows the team to scale the team maturity curve faster, as implied in Tuckman's classic article 'Developmental sequence in small groups' [34]. Thus, changing the nature of the interaction between team members allows the team to shift through the forming-storming-norming-performing stages of team development at an accelerated pace, thereby increasing team productivity.

Project teams often get stuck at the forming or storming stages. In reality, very few teams make it through to the norming and performing stages. Through tracking issues and accompanying states of being, the software draws attention to patterns that cause the team to stagnate in a particular state of dysfunction. Identifying such patterns unequivocally identifies the stage of development a team has reached, and sets the basis for the team to begin to move away from such patterns to more desirable ones.

If there is a prevalent pattern of states that can be thought of as the outcome of the real-time dynamics of $U, M_{1}, M_{2}$, and $M_{3}$ - such as 'synthesising', 'reasoning', 'calmness', 'patience', and 'enthusiasm', punctuated only by instances of states such as 'haste' and 'fear', for example - this would seem to indicate that a team is operating at the norming stage. A norming stage is where the team has begun to develop and follow rational contracts to govern itself [31]. The software allows teams to become more aware of the patterns holding them up, allows them to begin to surface and address issues in a safer way, provides insights into the particular circumstances that typically cause such patterns of dysfunction, suggests numerous ways in which to begin to move to better patterns of functioning, and allows tracking and shifting of such patterns in real time.

The 50 leaders (approximately), divided into seven project teams, worked on strategic hospitalbased initiatives, and were asked to track team dynamics on a regular basis. In fact, they were given the choice whether or not to track team dynamics. While individuals in those teams who chose to use the tool reported increased sensitivity to feelings-based team dynamics, one of the research questions is whether the use of the tool actually increased productivity at the team level. Results from a simulated subarctic survival exercise [18] through which each of the seven teams went indicated this to be the case. In this simulation, involving a plane crash, each team member was individually asked to rank 15 items necessary for their survival. The team then collectively ranked the same items. If the team score is better than the best individual score, then synergy is deemed to have occurred. If the team score is worse than the best individual score, then synergy is deemed not to have occurred. A lower score implies a higher degree of team synergy.

The investigation revealed a couple of clear correlations [4] between deeper sources of innovation and the overcoming of patterns, as suggested by Equation (1) and, specifically, by Equation (6). First, those teams whose use of the team dynamics tool was low exhibited lower synergy, as suggested by Figure 4 . Second, those teams whose use of the team dynamics tool was at the ideal level, even for a period of time, registered a higher degree of synergy, as measured by the survival exercise. This is likely because becoming aware of, calling out, and acting on negative dynamics in real time positively impacts the nature of interaction between the team members. In other words, and ex post facto, the untransformed set Integral $_{U}$ began to move towards the transformed set Integral $_{T}$ as specified in Equation (6). Synergy, which is contained in this equation, was positively affected. It is also likely that the very act of becoming aware of, and calling out, positive dynamics tended to reinforce them.

Figure 4 summarises the correlation or relationship between teams who more actively used the team dynamics tool, and their level of synergy, as measured by the subarctic survival simulation: 


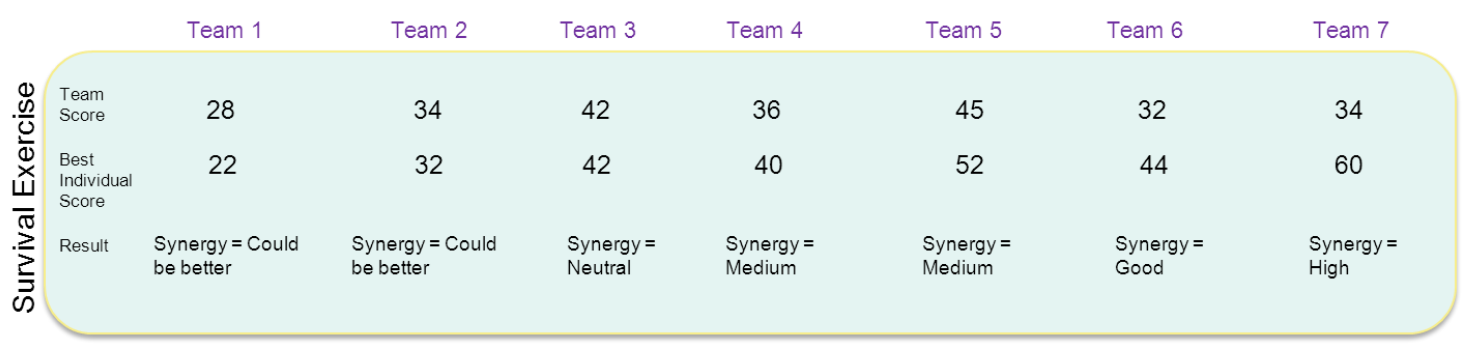

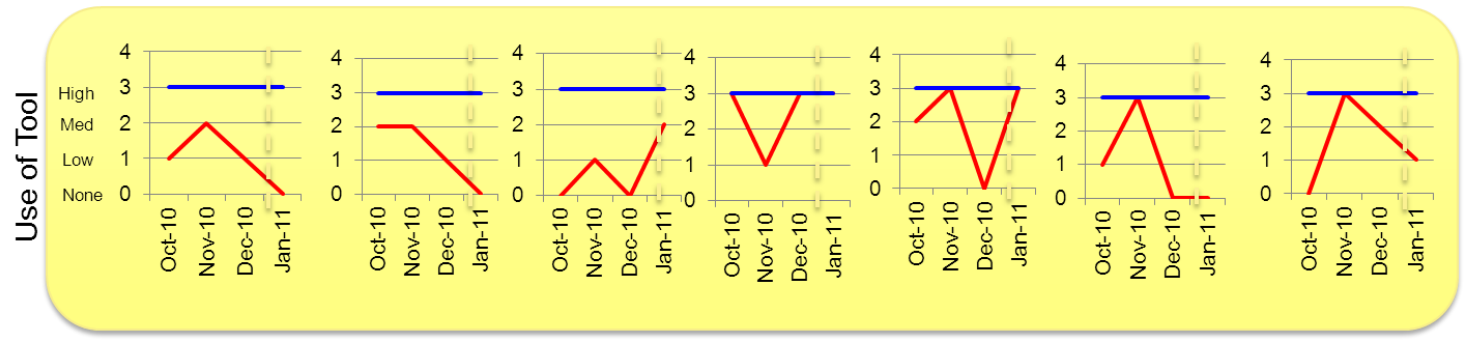

Figure 4: Stanford Hospital \& Clinics and author's correlation of Emotional Intelligence Builder
software with increased team synergy

As can be seen, a couple of correlations are suggested. First, those teams whose use of the team dynamics tool was low (max level = medium, in the yellow graphic in Figure 4 ) exhibited lower synergy. Note that the use of the tool was measured over a four-month period on the dates indicated on the $x$-axis of the graphs. Second, those teams whose use of the team dynamics tool was at the ideal level (indicated by the blue line in the yellow graphic in Figure 4), even for some period of time, registered a higher degree of synergy, as measured by the survival exercise in the blue graphic in Figure 4. This was in part, at least, reportedly caused by their subsequently internalising the mechanisms learned through using the Emotional Intelligence Builder tool. Note that the formulation of the mathematical equations of innovation is currently of an exploratory nature. Thus the aforementioned correlations are qualitative as opposed to quantitative, and therefore do not, at this stage, require $p$-tests to be performed [3].

\section{WORK WITH THE PEDIATRIC INTENSIVE CARE UNIT - DEPARTMENTAL LEVEL}

Following the programme with the Leadership Academy, the leadership teams of several departments at Stanford University Medical Center (discussed here as ex post facto case research) engaged in multi-month projects to improve the management of their departments through the activation of additional sources of innovation. This section covers a representative piece of work conducted at the Pediatric Intensive Care Unit (PICU) at Stanford University's Lucille Packard Children's Hospital.

The PICU is an area within the hospital specialising in the care of critically ill infants, children, and teenagers. Because of the acute illness of PICU patients and the risk of life-threatening complications, the ratio of professionals to patients is generally higher than in other parts of a hospital. Complex technology and equipment (such as mechanical ventilators and patient monitoring devices) are often in use, and make for a more complex socio-technical environment. This means that the PICU generally has a larger operating budget than other areas of a hospital, and typically has more organisational development-related issues. As related by the leaders in dialogue with the primary author, the PICU could do with improved employee engagement and patient satisfaction scores. Studies indicate a direct correlation between these scores and future revenues [1].

The Director of the PICU had heard about the approach employed by the first author in helping leaders and departments, and wanted to engage in a three-month project. The project kicked off with a one-day training and orientation, in which the PICU Leadership Team was initiated into the world of fractals and innovation, and the real choice they had in accepting or changing their operating reality by making small personal changes in their active states of being, and in alignment with Equations (1) and (3) to (6). 
The one-day orientation grounds participants in self- and team-awareness. The day starts with some hypothetical exercises. A typical scenario may be the following: "Imagine you are all working for a very demanding and self-serving boss who wants more than anything else to meet their own goals, regardless of what that implies for their staff. Now imagine there was a fire in one of the local schools and many children are being rushed to be treated at the hospital. The PICU rapidly fills up, and stress levels amongst nurses quickly escalate. This affects decision-making, and continues to put the department and hospital at higher than normal risk. You have to get the environment back to normal as quickly as possible".

The participants all have access to the Emotional Intelligence Builder web-based environment (as described in the previous section). and record their perceptions of self and of others in the leadership team. The framing questions to record such observations may be of the following nature: "What are you experiencing? What do you perceive your colleagues on this team are experiencing? What is the nature of decision-making? How are you handling conflict as a team?". These questions are consistent with the instructions in Figure 3, and allow individuals and teams to focus on how the sources of innovation, as depicted in Equation (1), may be manifesting practically. All entries are anonymous, but contribute toward a single 'team dashboard', projected in real time. There are over 40 emotion-based patterns to choose from, as illustrated in Figure 1, ranging from lethargy, boredom, fear, anger, and frustration to joy, courage, synergy, and so on. Figure 5 illustrates representative information captured for the team.

Since such patterns are visceral, they tend to influence the working culture of the team much more powerfully than ideas or thoughts in the short term [16]. From a research perspective, the intensity and duration scales depicted in Figure 5 give insight into the nature of the patterns currently in play. If a higher intensity pattern is of an untransformed nature, the likelihood of $M_{1}, M_{2}$, and $M_{3}$ being able immediately to influence innovation is lower. If a higher duration pattern is of an untransformed nature, the likelihood of $M_{1}, M_{2}$, and $M_{3}$ being able to influence innovation in the short to medium term is lower. As participants continue to reflect on the questions in the context of the hypothetical situation, definite patterns emerge that are immediately reflected on the summary team screen. After 30 minutes of entry, the team then switches gear and begins to analyse this data. What is the direction-pattern indicating about the situation? Since patterns repeat themselves on different scales - that is, on the individual, team, and departmental level - what is the pattern going to indicate about how the department is likely to operate in this scenario? Different patterns indicate different operating realities, and by looking at a pattern, one can tell the nature of the operating reality and the stage of development of the team/department quite conclusively [5].

Now that the team has been introduced to the idea of patterns, self- and other-awareness, and the fractal imprint, the second half of the day switches to real scenarios. Typical scenarios latch on to real and critical projects or changes that the department is currently going through, or the nature of leadership that is being felt by the team. For this first-day orientation, the PICU Leadership Team decided to focus on a department restructuring that they were currently going through.

Usually, analysing the patterns being displayed concludes the first day. This offers a quick visceral insight into the nature of the problems and issues, and the summary gestalt being experienced because of the restructure. Typical questions into which participants gain insight include: "What are people confident about? What do they fear are the big obstacles? Are there personalities coming in the way of the work? What is the general mood in the environment? How much conflict is being swept under the carpet to perhaps surface in other and often more destructive ways?" These questions also give insight into any shifting nature of innovation, as depicted in Equation (1) and Equations (3) to (6).

As with most teams, the summary pattern being displayed indicated that the team was somewhere between the forming and storming stage [34], even though the leadership team had worked together for over a year, and the project was already a few months past the launch phase. This was a less than positive prognosis, and quickly generated a number of hypotheses about how employee engagement and patient satisfaction scores could improve. 


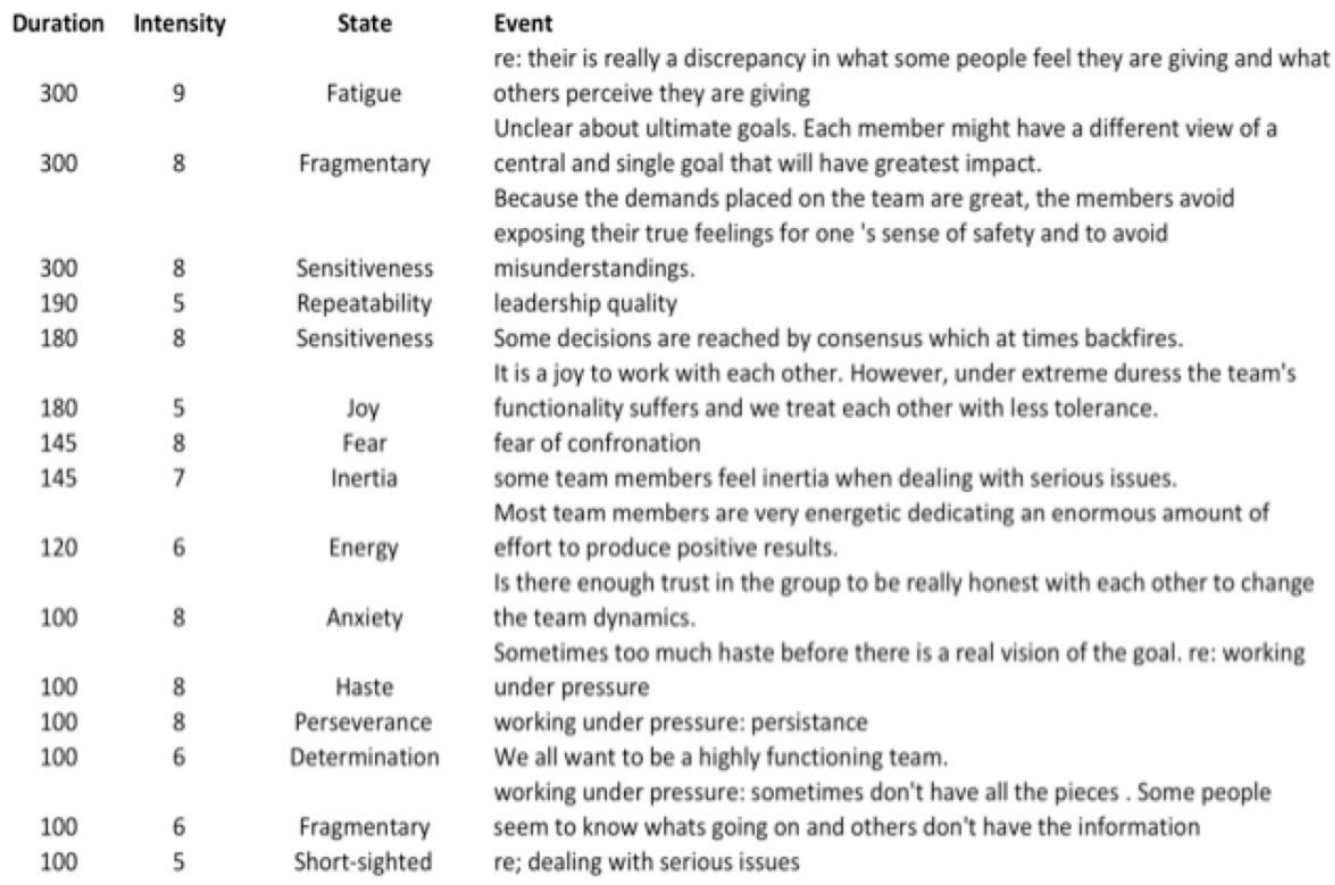

Figure 5: Representative information captured for the team

At the end of the day, the team contracted to use the Emotional Intelligence Builder software as a service (SaaS) environment on a regular basis. They were advised to engage with the SaaS environment every time they had a team meeting or an interaction with one another. It typically takes only a few minutes to enter one's feelings and thoughts. It was agreed to meet at the end of the month and analyse the data together. The first author, as field researcher, also entered into a coaching arrangement with several of the leaders to help them see the reality they were creating through who, and what, they were, or were not, being and doing.

The PICU leadership team went through a few iterations of this cycle of the team entering observations in the software tool for a month and being mentored by the first author, based on analysis of the data, and then collectively analysing the patterns and their implications. This approach forced the leaders to see things that they would not normally focus on, and forced them to take actions they would not earlier have taken. As a result, based on the observations of the first author, in conversation with the PICU leadership team, the team maturity improved, as did the active management of the restructuring project. The foundation for sustainably improving employee engagement and patient satisfaction scores was therefore put in place through more systematically exercising several additional sources of innovation. These additional sources, which surfaced in conversation with the PICU leadership team, included 'team synergy' (from the set of system nurturing), 'objective decision-making' (from the set of system knowledge), and a higher degree of 'diligence' (from the set of presence), as summarised in Section 2. These in turn, and ex post facto, further activated the transformations captured by Equations (6), (5), and (3) respectively, to put in place a more sustainable reality of innovation.

\section{THE FRACTAL ORGANISATION FIELD GUIDE}

Following a multi-year investigation, the first author, as field researcher and director of the Organizational Development Department, sought to create a practical fractal-based methodology that hinged on the physical, the vital, and the mental, and subsequently embarked on a multi-year journey to create a field guide to be used by any department or corporation seeking to bring about sustainable innovation. 
This field guide was a key part of the peer-reviewed The fractal organization [22], with a foreword by Dean Dipak Jain of Kellogg Graduate School of Management, INSEAD - a premier graduate business school with several campuses around the world, and Sasin Graduate Institute of Business Administration in Thailand. Certain sections of the foreword, reproduced below, reinforce and support the validity of the research approach behind the mathematical equations for innovation being researched ex post facto through their applicability in the Stanford case study.

"This is a different kind of business leadership book.

It is, in some sense, a book about archeology and architecture on a cosmic and commercial scale...

...This is a book that explores what might be called "the wholeness of the parts" in an effort to demonstrate the significance of patterns-specifically, fractal patterns-in our lives.

... a fresh and daring perspective in an attempt to bridge the science of fractals with the larger world comprised of these smaller pieces.

...From individual to institution, there are profound connections.

...Malik explores these relationships at the micro level and endeavors to highlight their accumulated power at the macro level too.

...Malik invites the reader on a journey into the fractal realm to show how patterns at this level are reproduced and reverberate throughout our social and cultural institutions, including our organizations..."

Further, this book, encapsulating the practical field guide (itself an outcome and culmination of the journey at the case organisation, and the fractal-based theoretical foundation) is summarised by the Table of Contents (TOC) of The fractal organization in Figure 6, 'The fractal organization TOC, illustrating field guide and power of patterns'. The TOC suggests the power of small patterns, from a theoretical and practical perspective, in shaping entire organisational realities. These, from a research perspective, have been an important input in creating the mathematics of organisational innovation being researched ex post facto through their applicability in this case research.

The book was endorsed by several of the Stanford University Medical Center leaders, as summarised in Figure 7, 'Selected quotes from the endorsement section of The fractal organization by Stanford University Medical Center leadership'. These endorsements further reinforce the general research that stands behind the formulation of the equations, and the likely validity of their applicability in framing organisational innovation.

\section{CONCLUSION}

The framing and subsequent enhancement of innovation, as captured by Equations (1) to (6), began with a small group - the core OD Team - and progressed to a series of courses offered through the Stanford Center of Education and Professional Development. This phase of the work is summarised in Section 3, and highlights the overcoming of restrictive patterns of innovation at the individual level.

A leadership cadre subsequently used the same programme over a six-month period. As part of the ex post facto case research, this study gives insight into overcoming restrictive patterns of innovation at the team level, and is summarised in Section 4.

The work with the leadership cadre led to several departmental leadership teams also participating in a multi-month innovation engagement to improve performance at the departmental level. This phase of the work was summarised in Section 5, and essentially highlights, ex post facto, the overcoming of restrictive patterns of innovation at the departmental level. 


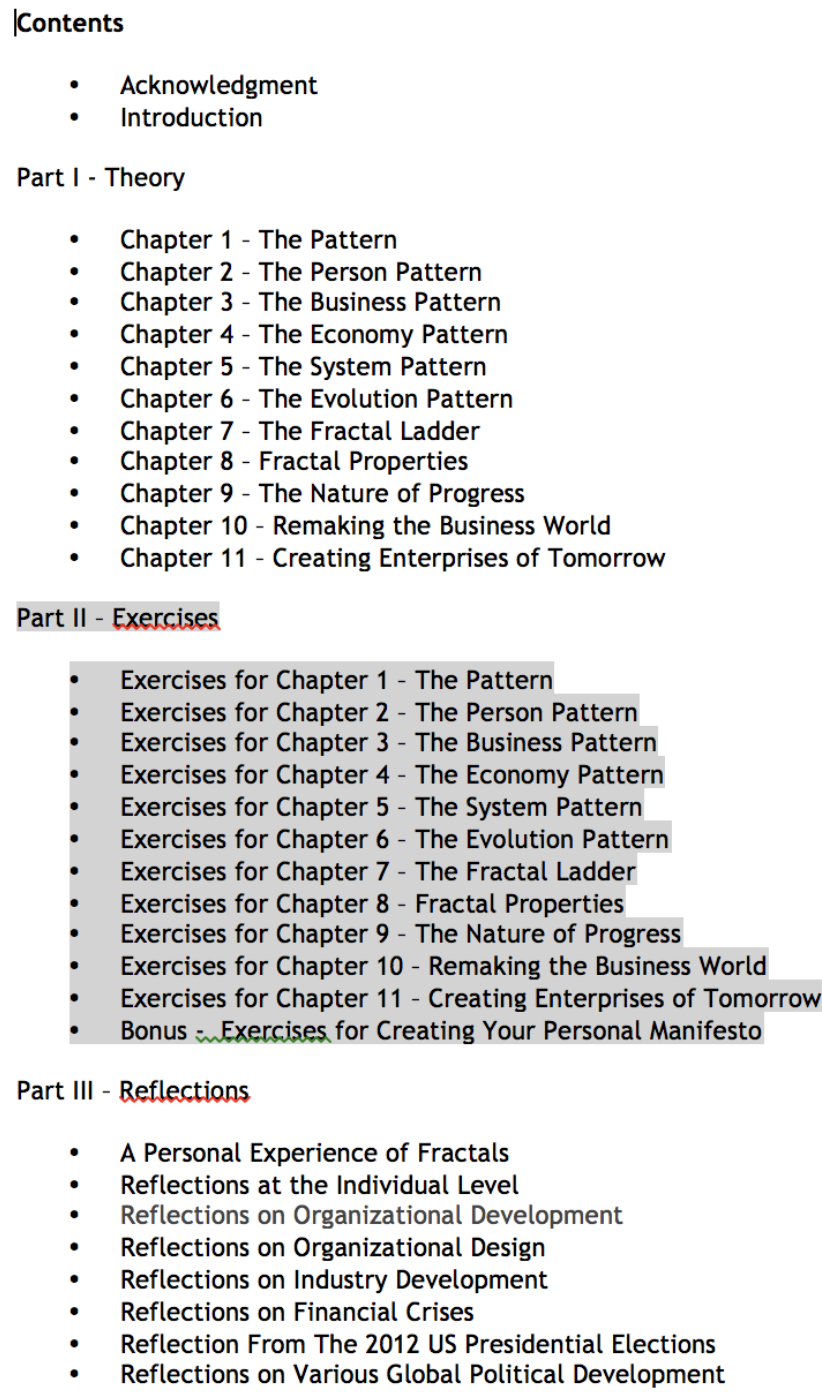

Figure 6: The fractal organization TOC, illustrating field guide and power of patterns

This work culminated in a practical field guide, positioned to be used at variable scales of complexity, that was published in The fractal organization [22], and that sought to capture key underlying aspects of the multi-year experiment. This field guide offers practical ways to overcome restrictive patterns of innovation at multiple scales, and has informed the development of Equations (1) to (6), which have been used subsequently in the ex post facto case research to frame the management of organisational innovation covered in this paper.

This work had a degree of success as captured and reported by several sources. In 2011, in particular, a Forbes reporter [30] wrote an article, 'Mindfulness as a tool of organizational and social change', that captured the essence of this work. Furthermore, the Society of Human Resource Management produced a report [29], 'Advancing sustainability: HR's role', in which an expert view on changing the culture at Stanford University Medical Center (SUMC) was provided by Mr Prigge, Director of Organizational Development at Stanford University Medical Center, on the fractal effects of small changes at the individual level on larger issues of the discipline of sustainability. 
I've been impressed with Pravir's work using Fractal thinking specifically as it relates to managing change. The methodology in The Fractal Organization: Creating Enterprises of Tomorrow opens up perspectives not normally considered, that can lead to highly accelerated adoption of the change process.

Laura Gottlieb, Director - Enterprise Learning \& Development, Lucille Packard Children's Hospital at Stanford

By calling attention to the fractal patterns that reside within and all around us, Pravir Malik has given us a set of keys to unlock our inner potential, enabling us to more fully partner with "Progress" and ascend the fractal ladder. ...With great depth and heart, Malik goes beyond merely referencing this powerful quote, he offers readers a simple formula (via the physical, vital and mental fractal) to BE the change. This book makes the connection between the individual and the world we each helped create, while at the same time elevating readers' consciousness to envision and co-create an even brighter future.

\title{
Dale Spartz, Ph.D., VP - HR, Stanford Hospital \& Clinics
}

\begin{abstract}
..."The Fractal Organization: Creating Enterprises of Tomorrow" is quite different. It builds on the previous work of the author (Connecting Inner Power with Global Change: The Fractal Ladder (Malik, 2009) and is based on a solid theoretical foundation from the fields of psychology, economics, anthropology, and behavioral science. It offers a unique view of change and system patterns. This book reviews the theory behind the Fractal Ladder, provides exercises at an individual or group level for reflection and learning, and provides reflections \& analysis for individual, group, and organizational levels...The book is not intended to be an "easy read." One needs to spend time understanding the theory and the "fractal architecture" of physical orientation, vital orientation, and mental orientation....
\end{abstract}

\section{Figure 7: Selected quotes from the endorsement section of The fractal organization by Stanford University Medical Center leadership}

In the director's words [29]: “Change programs are often initiated at the policy level. Such top-down orchestration is important, but it does not always work. To make change sustainable, regardless of the type of change, it would be most effective to supplement top-down change with actual shifts in attitudes, behaviors and even perceptions. This is what I am focusing on at SUMC. To make it happen, we have initiated a series of organizational interventions and courses that focus on shifting the individual point of view from the physical to the vital to the mental. Such interventions and courses focus on team-building, conducting crucial conversations, conflict resolution, coaching, among others. These are critical in shifting individual behavior. The 'physical' refers to old, established ways of doing things that have often outlived their utility but continue to be followed because of force of habit. The 'vital' refers to a lot of experiment, often led by feeling and emotion, and is critical as an organization tries to break away from established ways of doing things. The 'mental' refers to well-thought-out and holistic ideas that, in fact, must become the engine of decisionmaking, as opposed to habit or emotion. To build sustainable organizations, it is essential that employees begin to operate at the mental level. But equally the well-thought-out ideas have to be supported by the vital-the emotion and feeling-and the physical-the past capital and infrastructure that are the result of the organization's historical success."

The director's assessment of the fractal effects of small changes reinforces, ex post facto, the essential fractal structure of Equations (1) to (6), and their applicability as a framework for managing organisational innovation by continually making small shifts in attitudes, behaviours, and perceptions, as researched in this paper.

The elaboration of distinct sets of sources of innovation - the $\mathrm{x}_{\mathrm{u}}$ sets at layer $\mathrm{U}$, the variation on $S_{\text {System }_{x}}$ sets at Layers $M_{1}$ and $M_{2}$ and the resultant $x_{t}$ sets, and the conditions for their activation as laid out in Equation (1) and Equations (3) to (6) as summarised in Section 2 - is a unique way to 18 
frame the management of organisational innovation. This ex post facto case research at the individual, team, and departmental levels (covered by Sections 3, 4, and 5) implies that the application of these equations to a complex adaptive system such as Stanford University Medical Center offers a way to overcome restrictive patterns of innovation, and thereby allows deeper sources of innovation to become active.

Further details on the multi-year journey at Stanford and its spread into other areas are captured by the primary author on Medium [24].

\section{REFERENCES}

[1] Al-Mailam, F. 2005. The effect of nursing care on overall patient satisfaction and its predictive value on return-to-provider behavior: A survey study, Quality Management in Health Care 14, 2, 116-120.

[2] Anderson, P. 1972. More is different. Science, New Series, Vol. 177, No. 4047. (Aug. 4, 1972), pp. 393396.

[3] Cooper, D. \& Schindler, P. 2014. Business research methods. New York: McGraw-Hill/Irwin.

[4] Aurosoorya. 2011. The mirror effect: Experiments in accelerating team development. http://blog.aurosoorya.com/2011/08/06/the-mirror-effect-experiments-in-accelerating-teamdevelopment/Accessed: 01. Oct. 2017

[5] Aurosoorya. 2015. Next generation human capital systems. http://blog.aurosoorya.com/2015/02/23/next-generation-human-capital-enterprise-systems/ Accessed: 01. Oct. 2017

[6] Bavelier, D., Green, C., Pouget, A. \& Schrater, P. 2012. Brain plasticity through the lifespan: Learning to learn and action video games. Annual Review of Neuroscience. 35: 391-416.

[7] Chan, S. 2001. Complex adaptive systems. MIT. ESD-83 Research Seminar in Engineering Systems.

[8] Deep Order Technologies Team Analytics. 2016. Deep order technologies. http://www.deepordertechnologies.com/technology Accessed: 01. Oct. 2017.

[9] Dodder, R. \& Dare, R. 2000. Complex adaptive systems and complexity theory: Inter-related knowledge domains. MIT. ESD.83: Research Seminar in Engineering Systems.

[10] Martin, W \& Ott, M. 2013. The Cosmic View of Albert Einstein: Writings on Art, Science and Peace. Sterling Publishing, New York, NY.

[11] Felicia, P. 2011. Handbook of research on improving learning and motivation through educational games: A multi-disciplinary approach. Hershey: IGI Global.

[12] Feynman, R.P. 1985. QED: The strange theory of light and matter. New Jersey: Princeton University Press.

[13] Gell-Mann, M. 1994. The quark and the jaguar: Adventures in the simple and the complex. Nature, 369(6481), pp. 529-529.

[14] Gell-Mann, M. 2003. Regularities and randomness: Evolving schemata in science and the arts. Art and complexity. pp. 47-58.

[15] Gershenson, C. 2013. Living in living cities. Artificial Life, 19(3-4): 401-420.

[16] Goleman, D. 2005. Emotional intelligence: Why it can matter more than IQ. New York: Bantam Books.

[17] Hawking, S. 1988. A brief history of time. New York: Bantam Books.

[18] Human Synergistics. 2013. Sub arctic survival exercise. http://www.subarcticsurvival.com/overview/. Accessed: 01 Oct. 2017.

[19] Lencioni, P. 2002. The five dysfunctions of a team: A leadership fable. San Francisco: Jossey-Bass.

[20] Malik, P. 2009. Connecting inner power with global change: The fractal ladder. New Delhi: Sage Publications.

[21] Malik, P. 2011. Redesigning the stock market: A fractal approach. New Delhi: Sage Publications.

[22] Malik, P. 2015a. The fractal organization. New Delhi: Sage Publications.

[23] Malik, P., Pretorius, L. \& Winzker, D. 2015. A mathematical basis of innovation. Conference Proceedings IAMOT 2015. Paper 090

[24] Malik, P. 2015b. OD experiments at Stanford University. https://medium.com/@PravirMalik/some-odexperiments-at-stanford-university-medical-center-i-479f78608eda\#.nislun540. Medium.

[25] Malik, P. 2017. Development and evaluation of a framework for an engine of innovation in complex adaptive systems. PhD thesis, University of Pretoria, Pretoria, http://hdl.handle.net/2263/62779. Accessed: 01. Oct. 2017

[26] Malik, P., Pretorius, L. \& Winzker, D. 2017. Qualified determinism in emergent technology complex adaptive systems. Conference Proceedings IEEE-TEMSCON 2017. pp 113-118.

[27] Prigogine, I. 1997. The end of certainty. New York: Free Press.

[28] Sevilla, C., Ochave, J., Punsalan, T., Regala, B. \& Uriarte, G. 1992. Research methods. Quezon City: Rex Book Store.

[29] SHRM. 2011. Advancing sustainability: HR's role. https: / /www.shrm.org/resourcesandtools/tools-and-samples/toolkits/documents/110066_advsustainhr_fnl_full.pdf. Accessed: 01 Oct. 2017. 
[30] Skibola, N. 2011. Mindfulness as a tool for organizational and social change. Forbes. http: / /www.forbes.com/sites/csr/2011/02/01/mindfulness-as-a-tool-for-organizational-and-socialchange/. Accessed: 01 Oct. 2017.

[31] Stein, J. 2016. Using the stages of team development. Boston: MIT. http://hrweb.mit.edu/learningdevelopment/learning-topics/teams/articles/stages-development-topics/teams/articles/stagesdevelopment.

[32] Stewart, lan. 2002. Does God play dice? The new mathematics of chaos. Oxford: Blackwell Publishing.

[33] Thomas, J. \& Zaytseva, A. 2016. Mapping complexity/Human knowledge as a complex adaptive system. Complexity, 21: 207-234. doi:10.1002/cplx.21799.

[34] Tuckman, B. 1965. Developmental sequence in small groups. American Psychological Association Psychological Bulletin, 63(6): 384-99.

[35] West, G. 2014. Scaling: The surprising mathematics of life and civilization. Santa Fe Institute Bulletin 28(2).

[36] West, G. 2017. Scale. New York: Penguin Press. 